\title{
Histological and Morphometric Examination of the Testes of Boars and Male Pigs Immunocastrated with Improvac ${ }^{\circledR}$
}

\author{
Slobodan Stojanovic', Gordana Uscebrka', Dragan Zikic² \& Marina Stukelj
}

\begin{abstract}
Background: Surgical castration of male piglets is frequently performed to prevent development of an unpleasant taste and flavor in meat of mature males, called boar taint. Disadvantages of this method are primarily related to the lower feed efficiency of surgically castrated male pigs, production of less lean meat and more fat on the carcasses than boars, as well as ethical issues associated with the procedure itself. Immunocastration is a method of immunization against gonadotropinreleasing hormone $(\mathrm{GnRH})$ and represents an alternative to avoid boar taint. This study aimed to examine the influence of immunocastration on histological and morphometric parameters of male pig testes.

Materials, Methods \& Results: A total of 60 crossbred male pigs selected from 30 litters were assigned to two experimental groups: boars $(\mathrm{n}=30)$ and immunocastrated males $(\mathrm{n}=30)$. Two doses $(2 \mathrm{~mL}$ per dose $)$ of the Improvac ${ }^{\circledR}$ vaccine were $^{2}$ given to pigs scheduled for immunocastration. The first injection was given at 10 weeks of age, and the second injection at 20 weeks of age. All pigs were fed the same commercial diet ad libitum, group-housed (15 pigs/pen) and slaughtered at 25 weeks of age. After slaughter, testicle weights were measured and samples of testicular tissue were taken for histological examination. Immunocastrated males had lower testicle weights than boars $(P<0.01)$. Furthermore, structural damage to the germinal epithelium was visible in the seminiferous tubules of vaccinated pigs. In the testicular interstitium of these animals, the number of Leydig cells were reduced; almost all of these cells exhibited different degrees of abnormality. Moreover, the diameter of the seminiferous tubules and thickness of the germinal epithelium were greater $(P<0.01)$, and the percentage of germinal epithelium in the seminiferous tubules as well as percentage of blood vessels in the interstitium were higher in boars as compared to immunocastrated males $(P<0.01)$. Seminiferous tubule lumen diameter was greater $(P<0.01)$, and percentage of lumen in the seminiferous tubules as well as percentage of non-blood vessel tissue in the interstitium were higher in immunocastrated males than boars $(P<0.01)$.

Discussion: Vaccination against GnRH, as used in this study, reduced the synthesis of follicle-stimulating hormone (FSH) and luteinizing hormone (LH), which led to decreased spermatogenesis and androgen production. The smaller diameter of the seminiferous tubules as well as germinal epithelium thickness, and lower percentage of germinal epithelium in the seminiferous tubules in immunocastrated males could be explained by reduced FSH synthesis, and subsequent reduced spermatogenesis. The described testicular malformations in immunocastrated animals caused their lower weight, and greater diameter of the lumen as well as a higher lumen percentage of the seminiferous tubules. The vaccine reduced LH synthesis, so the Leydig cells of immunocastrated animals had lower activity than the same cells in boars. The percentage of blood vessels in the interstitium of boar testes was higher, which was related to the blood supply required for normal function of Leydig cells. It was concluded that differences existed in the structure of the seminiferous tubules and interstitium between boars and immunocastrated males, which were reflected in values of the examined histological and morphometric parameters. The development of these differences was related to the inhibition of GnRH synthesis and secretion.
\end{abstract}

Keywords: pig, immunocastration, testicular morphology. 


\section{INTRODUCTION}

Immunocastration represents one way to avoid surgical castration of male piglets with the aim of preventing the development of odour-taint in their meat. For a long time, surgical castration was the standard method used for this purpose despite its drawbacks. In comparison with boars, surgically castrated males have lower feed efficiency, less lean meat and more fat in their carcasses [30]. Surgical castration has also been criticized from an animal welfare viewpoint $[3,9,24,25]$.

Immunocastration with the Improvac ${ }^{\circledR}$ vaccine delivers a vaccination against gonadotropin-releasing hormone $(\mathrm{GnRH})$, which leads to a reduction in the release of follicle-stimulating hormone (FSH) and luteinizing hormone (LH) $[21,27]$. These processes change testicular function and reduce the synthesis of androstenone, a steroid primarily synthesized in the Leydig cells [17]. In addition to androstenone, skatole is also responsible for boar taint [5], and since their metabolism could be related [1], it was reported that immunocastration is effective in diminishing boar taint $[13,15]$.

One alternative method of preventing the development of odour-taint in meat from male pigs is based on the use of genetic markers in the selection of pigs that are free of boar taint, but further study is required to complete this examination and produce useful results [23,32].

The main objective of this study was to examine the histological and morphological changes in porcine testicular tissue caused by immunocastration with Improvac ${ }^{\circledR}$.

\section{MATERIALS AND METHODS}

\section{Animals and experimental design}

A total of 60 crossbred male pigs (crossbred Landrace/Yorkshire sows $\times$ crossbred Duroc/Pietrain boars) selected in the first week of life from 30 litters were assigned to two experimental groups: boars ( $\mathrm{n}=$ $30)$ and immunocastrates $(\mathrm{n}=30)$. Two doses $(2 \mathrm{~mL}$ per dose) of the Improvac ${ }^{\circledR}$ vaccine $^{1}$ were given to pigs scheduled for immunocastration. The first injection ( $2 \mathrm{~mL}$ ) was given at 10 weeks of age, and the second injection ( $2 \mathrm{~mL})$ at 20 weeks of age. Pigs in each experimental group were group-housed (15 pigs/pen), fed the same commercial diet ad libitum and kept under identical conditions. All the pigs were slaughtered at 25 weeks of age and testicular weight was recorded.

\section{Histological analysis}

Histological examination of testicular tissue from boars and immunocastrates was performed. Tissue samples were initially fixed in $10 \%$ buffered formalin solution, followed by dehydration, clearing, embedding in paraffin and cutting into serial 5 - $\mu \mathrm{m}$ thick sections using a microtome, and subsequently stained with haematoxylin and eosin $(\mathrm{H} \& \mathrm{E})[16,20]$.

The following histological parameters were recorded: diameter of the seminiferous tubules and the lumen of the seminiferous tubules (diameter was measured as the average of the longest lines drawn across the length and width of their cross-sections), thickness of the germinal epithelium, the percentage of germinal epithelium and lumen in the seminiferous tubules, and the percentage of non-blood vessel tissue and blood vessels in the interstitium. Microscopy was performed using a Leica DMLS ${ }^{2}$ light microscope with a Leica DC 3002 digital camera and the software package IM1000².

Percentage measurements were performed using the M42 testing system described elsewhere $[4,19,22,28]$, which basically consists of 21 line segments and 42 points in the testing area. For stereological analyses, the following formulas were used:

$$
\mathrm{P}(\mathrm{ge})=\frac{\mathrm{N}(\mathrm{ge})}{\mathrm{N}(\mathrm{st})} 100(\%)
$$

where: $\mathrm{P}(\mathrm{ge})$ - the percentage of germinal epithelium in the seminiferous tubules; $\mathrm{N}(\mathrm{ge})$ - the number of test points lying over the germinal epithelium; N(st) the number of test points lying over the seminiferous tubules;

$$
\mathrm{P}(\mathrm{l})=\frac{\mathrm{N}(\mathrm{l})}{\mathrm{N}(\mathrm{st})} 100(\%)
$$

where: $\mathrm{P}(\mathrm{l})$ - the percentage of lumen in the seminiferous tubules; $\mathrm{N}(1)$ - the number of test points lying over the lumen; $\mathrm{N}(\mathrm{st})$ - the number of test points lying over the seminiferous tubules;

$$
\mathrm{P}(\mathrm{nbv})=\frac{\mathrm{N}(\mathrm{nbv})}{\mathrm{N}(\mathrm{i})} 100(\%)
$$

where: $\mathrm{P}(\mathrm{nbv})$ - the percentage of non-blood vessel tissue in the interstitium; $\mathrm{N}(\mathrm{nbv})$ - the number of test points lying over the non-blood vessel tissue in the interstitium; $\mathrm{N}(\mathrm{i})$ - the number of test points lying over the interstitium; 


$$
\mathrm{P}(\mathrm{bv})=\frac{\mathrm{N}(\mathrm{bv})}{\mathrm{N}(\mathrm{i})} 100(\%)
$$

where: $\mathrm{P}(\mathrm{bv})$ - the percentage of blood vessels in the interstitium; $\mathrm{N}(\mathrm{bv})$ - the number of test points lying over the blood vessels in the interstitium; N(i) - the number of test points lying over the interstitium.

\section{Statistical analysis}

Statistical analyses were performed using oneway ANOVA and the post hoc Tukey's test to evaluate the effect of treatment on testes weight and morphometric parameters. Differences were considered significant at $P \leq 0.05$. Values are presented as mean \pm standard error of the mean. Statistical tests were carried out using Statistica for Windows, ver. 10.0.

\section{RESULTS}

Immunocastrated males had significantly lower testes weight $(P<0.01)$ compared to the boars (Table 1). Analysis of the photomicrographs showed differences in structure between the testes of boars and immunocastrates. Figures $1 \mathrm{~A}$ and $2 \mathrm{~A}$ show that in the fully developed germinal epithelium of boar testes, all developmental stages of spermatogenesis as well as spermiogenesis could be detected. Spermatozoa were observed in the lumen of the seminiferous tubules. All germinal epithelium cells were regularly shaped and structured. The germinal epithelium of immunocastrates (Figures 1B and 2B) was poorly developed. The lack of certain developmental stages of spermato- genesis in the seminiferous tubules was noticed. The degree of disruption in spermatogenesis varied from mild, as observed in a reduced number of germ cells in one layer, to serious damage such as complete absence of an entire layer or even several layers of germ cells, mainly spermatozoa, spermatids, secondary and primary spermatocytes. When spermatocytes were present, many of them were shrunken compared to the same cells in the testes of boars. The interstitium of boar testes contained numerous Leydig cells which had a regular shape and structure (Figure $3 \mathrm{~A}$ ). The number of Leydig cells in immunocastrates was reduced, and the morphology of these cells (Figure 3B) was changed (smaller cells of irregular shape with much less cytoplasm and poorly defined plasma membranes).

A greater seminiferous tubule diameter $(P<$ $0.01)$ was measured in boars compared to immunocastrates (Table 1). The diameter of the seminiferous tubule lumen was greater $(P<0.01)$ in immunocastrates (Table 1), while germinal epithelium thickness was greater $(P<0.01)$ in the seminiferous tubules of boars (Table 1).

The percentage of germinal epithelium in the seminiferous tubules (Table 1$)$ was higher in boars $(P<$ 0.01 ), while the percentage of lumen in the seminiferous tubules (Table 1) was higher in immunocastrates $(P<$ $0.01)$. In the interstitium of the testes, a higher percentage $(P<0.01)$ of non-blood vessel tissue was found in immunocastrates (Table 1), while the percentage of blood vessels was higher $(P<0.01)$ in boars (Table 1$)$.

Table 1. Effect of immunocastration on testicular weight and morphometric parameters.

\begin{tabular}{ccc}
\hline Weight and Morphometric Parameter & Boars & Immunocastrated males \\
\hline Testes weight $(\mathrm{g})$ & $574.67 \pm 9.26^{\mathrm{A}}$ & $234.83 \pm 5.26^{\mathrm{B}}$ \\
Seminiferous tubule diameter $(\mu \mathrm{m})$ & $202.88 \pm 4.75^{\mathrm{A}}$ & $160.11 \pm 5.34^{\mathrm{B}}$ \\
Seminiferous tubule lumen diameter $(\mu \mathrm{m})$ & $90.22 \pm 2.07^{\mathrm{B}}$ & $122.52 \pm 8.30^{\mathrm{A}}$ \\
Germinal epithelium thickness $(\mu \mathrm{m})$ & $112.66 \pm 4.61^{\mathrm{A}}$ & $37.59 \pm 5.02^{\mathrm{B}}$ \\
Germinal epithelium in seminiferous tubules $(\%)$ & $69.17 \pm 0.60^{\mathrm{A}}$ & $40.21 \pm 3.05^{\mathrm{B}}$ \\
Lumen in seminiferous tubules $(\%)$ & $30.83 \pm 0.60^{\mathrm{B}}$ & $59.79 \pm 3.05^{\mathrm{A}}$ \\
Non-blood vessel tissue in the interstitium $(\%)$ & $90.78 \pm 0.66^{\mathrm{B}}$ & $95.63 \pm 0.76^{\mathrm{A}}$ \\
Blood vessels in the interstitium $(\%)$ & $9.22 \pm 0.66^{\mathrm{A}}$ & $4.37 \pm 0.76^{\mathrm{B}}$ \\
\hline
\end{tabular}

Values are presented as mean \pm standard error of the mean. Means within a row with a different superscript letter differ significantly $(P<0.01)$. 


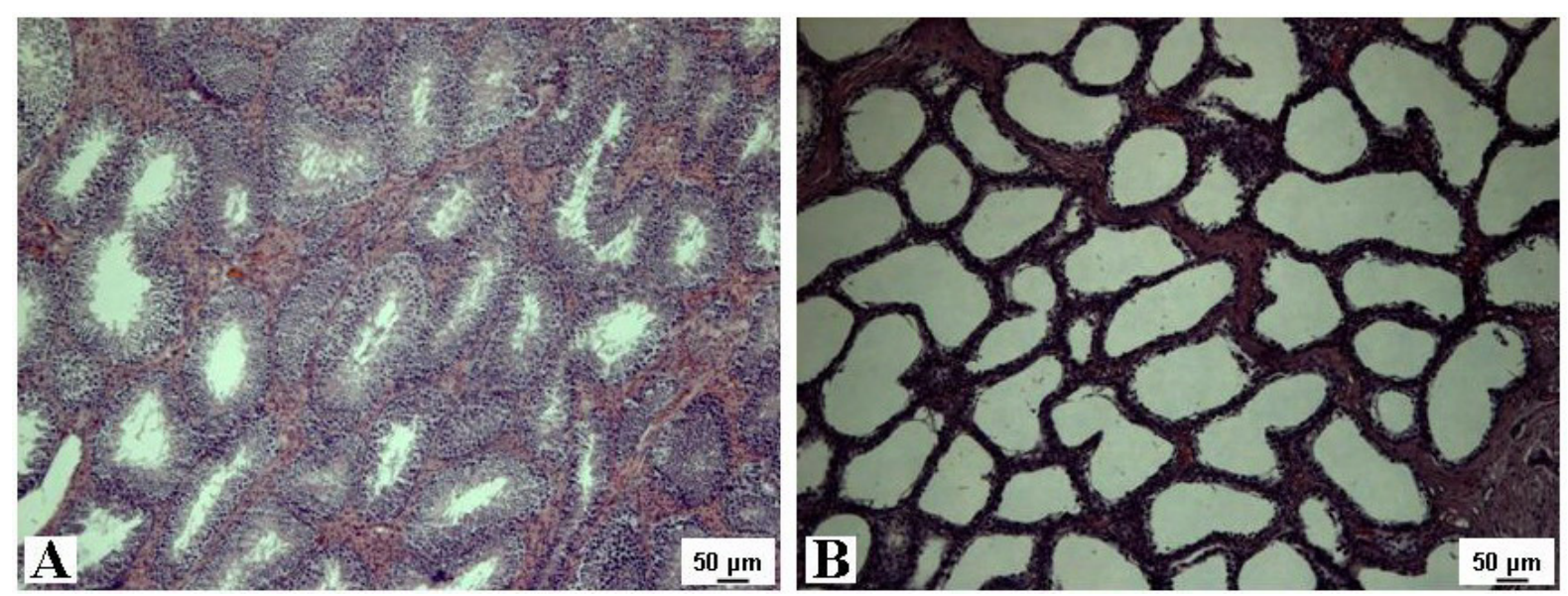

Figure 1. Cross section of testicular tissue showing the seminiferous tubules of boars (A) and immunocastrated males (B). H\&E staining. $[$ Scale bar $=50 \mu \mathrm{m}]$.
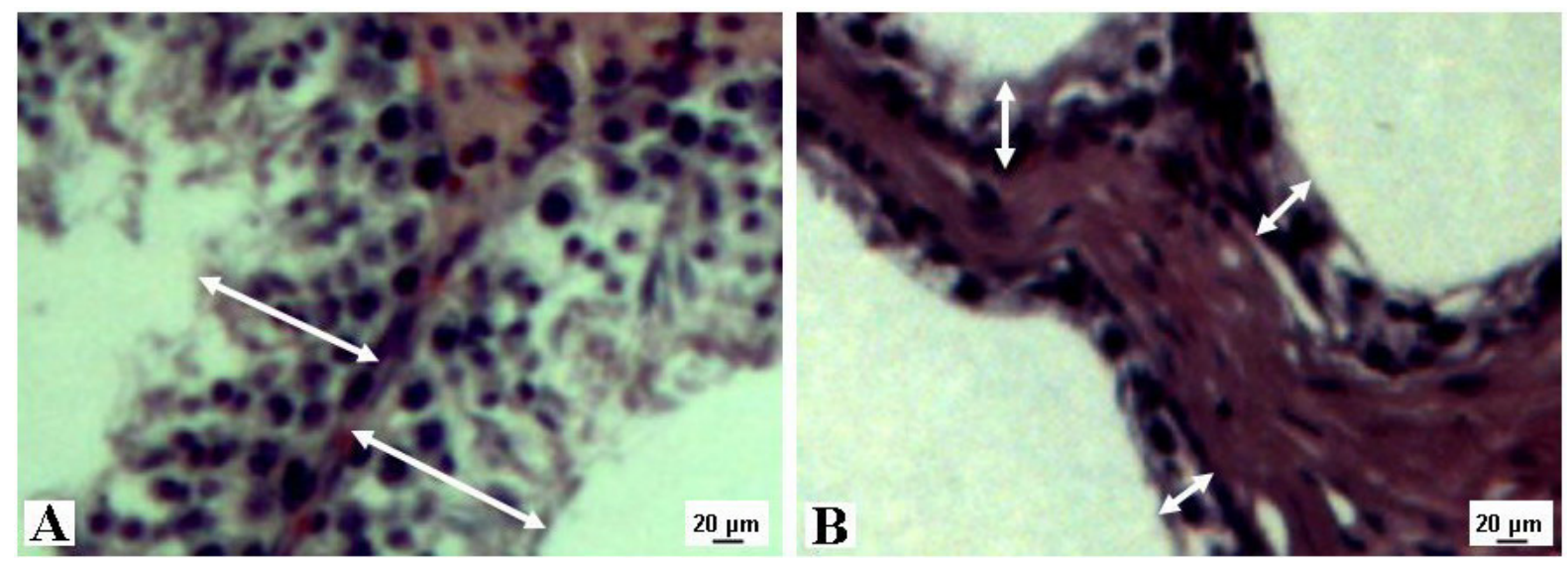

Figure 2. Testicle germinal epithelium thickness (arrows) of boars (A) and immunocastrated males (B). H\&E staining. [Scale bar $=20 \mu \mathrm{m}]$.
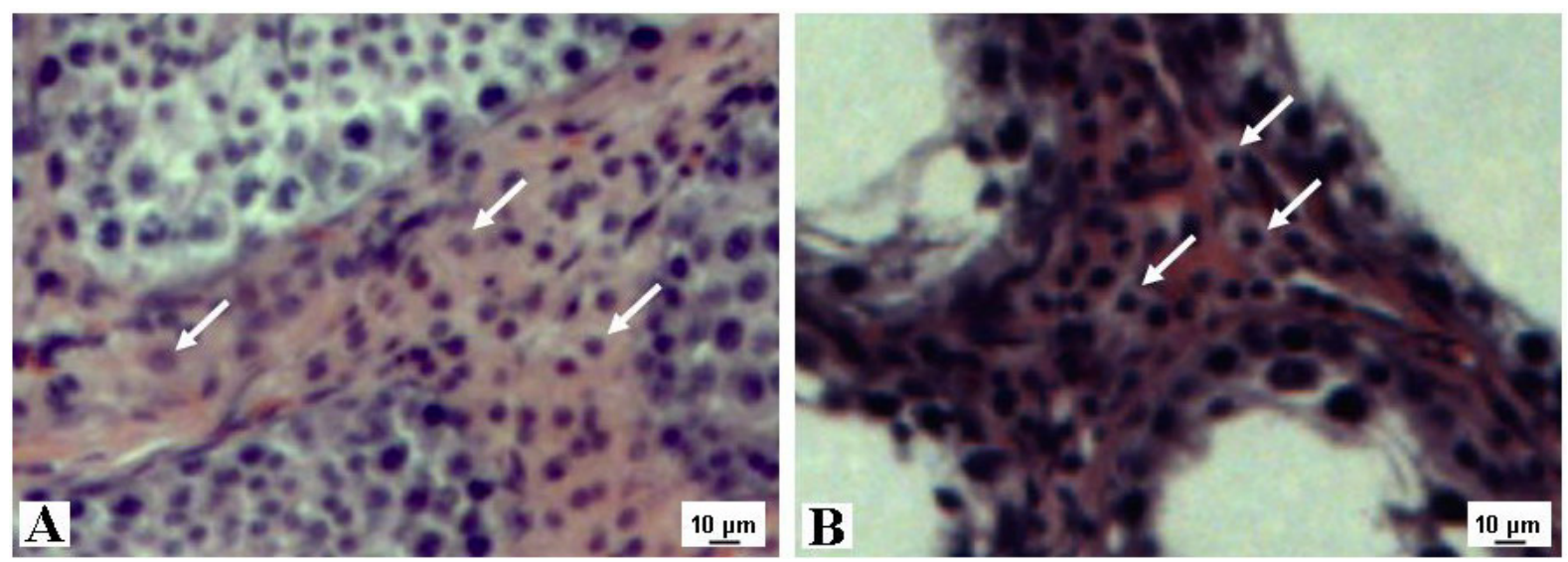

Figure 3. Testicular tissue cross sections of boars (A) and immunocastrated males (B) showing the Leydig cells (arrows) in the interstitium. $\mathrm{H} \& \mathrm{E}$ staining. [Scale bar $=10 \mu \mathrm{m}]$. 


\section{DISCUSSION}

Due to Improvac ${ }^{\circledR}$ vaccine administered in this study, which caused a reduction in FSH stimulation of spermatogenesis in the germinal epithelium of the seminiferous tubules as well as a reduction in LH stimulation of androgen production by Leydig cells [6,33], major changes in the morphology, histological structure and functionality of immunocastrated male testes occurred.

Analysis of the histological preparations obtained from immunocastrate testes revealed damage to the seminiferous tubule germinal epithelium. These changes were caused by influence of the vaccination on the reduced activity of gonadotropin cells in the adenohypophysis and consequently reduced production of FSH by these cells, which is required to the process of spermatogenesis [26]. This is in correlation with the previously described ultrastructure of gonadotropin cells in GnRH immunized boars, which exhibited a decreased number and size of large and small cellular granules, indicating altered cell morphology [10].

A greater or lesser degree of disruption to spermatogenesis, as detected in the seminiferous tubules of immunocastrates, has also been reported previously in immunocastrated mature boars [2], where the quantity of abnormal spermatozoa (abnormalities in head morphology, tail morphology, etc.) were also increased.

In the interstitium of the testes of immunocastrates, disrupted morphology and a reduced number of Leydig cells were related to reduced LH release caused by the vaccine. These processes reduced testosterone secretion by Leydig cells, which is in line with a previous study [31] where immunization against GnRH had a negative impact on plasma testosterone levels in the period after a second vaccination.

The greater thickness as well as higher percentage of germinal epithelium in the seminiferous tubules of boars, as compared to the immunocastrates that was reported in this study, was caused by the lower FSH levels $[10,11]$ and reduced spermatogenesis in the germinal epithelium of the seminiferous tubules of the immunocastrated males. In the testes of vaccinated animals, incomplete development or a complete lack of certain cells in the seminiferous epithelium as described earlier, was directly related to its reduced thickness. Furthermore, as the spermatogenic lineage in the seminiferous tubules of immunocastrates consisted of only a few cells, greater diameter of the lumen as well as a higher lumen percentage of the seminiferous tubules was detected. The greater diameter of the boar seminiferous tubules was explained by the fully developed cellular layers in the seminiferous epithelium. These results agreed with an earlier observation [2].

Lower testes weight was related to degenerative changes in the morphology of the testes caused by vaccination (smaller germinal epithelium thickness, smaller diameter of seminiferous tubules, etc.), which led to a decrease in their weight. Reduction in testes weight as well as reduction in the weight of accessory reproductive glands of immunocastrated males have been previously reported $[8,18]$.

The lower percentage of blood vessels in the interstitium of immunocastrated males can be related to the reduced function of the Leydig cells that produce less androgens due to decreased LH secretion. As Leydig cell function is associated with testicular interstitial blood flow [7,14], and function of these cells was reduced in immunocastrates, this could be related to poor blood supply to the Leydig cells and the detected lower percentage of blood vessels in the interstitium of immunocastrates. Reduced Leydig cell function was associated with the morphological changes described earlier. These findings are in accordance with data of other authors [12,29].

\section{CONCLUSIONS}

The results of this study indicated that immunocastration affects the histological and morphometric parameters of the testes. The vaccine disrupted spermatogenesis to varying degrees, which resulted in poor development of the germinal epithelium of the seminiferous tubules. The number of Leydig cells were reduced, while almost all of these cells had significant structural abnormalities. These processes caused differences in the observed parameters between the testes of boars and immunocastrated males, which were related to the inhibition of $\mathrm{GnRH}$ synthesis and secretion as a result of vaccination.

\section{MANUFACTURERS}

${ }^{1}$ Pfizer Animal Health. Parkville. Melbourne, Victoria, Australia.

${ }^{2}$ Leica Microsystems. Wetzlar, Hesse, Germany.

Funding. The study was supported by the Ministry of Education, Science and Technological Development of Serbia (Project No. TR 31034).

Ethical approval. The use of animals in this experiment was approved by the decision of the Ministry of Education, Science and Technological Development of Serbia (Decision No. 40100-9/2011 of 25 January 2011).

Declaration of interest. The authors report no conflicts of interest. The authors alone are responsible for the content and writing of the paper. 


\section{REFERENCES}

1 Babol J., Squires J. \& Lundström K. 1999. Relationship between metabolism of androstenone and skatole in intact male pigs. Journal of Animal Science. 77(1): 84-92.

2 Bilskis R. 2014. Immunocastration of young and mature boars with Improvac®. 63p. Kaunas, RL. Dissertação (Mestrado em Veterinary Sciences) - Veterinary Academy, Lithuanian University of Health Sciences.

3 Borell E.V., Baumgartner J., Giersing M., Jäggin N., Prunier A., Tuyttens F.A.M. \& Edwards S.A. 2009. Animal welfare implications of surgical castration and its alternatives in pigs. Animal. 3(11): 1488-1496.

4 Burity C.H.F., Pissinatti A. \& Mandarim-de-Lacerda C.A. 2004. Stereology of the liver in three species of Leontopithecus (Lesson, 1840) Callitrichidae - Primates. Anatomia Histologia Embryologia. 33(3): 183-187.

5 Claus R., Weiler U. \& Herzog A. 1994. Physiological aspects of androstenone and skatole formation in the boar: A review with experimental data. Meat Science. 38(2): 289-305.

6 Counis R., Laverriere J.N., Garrel G., Bleux C., Cohen-Tannoudji J., Lerrant Y., Kottler M.L. \& Magre S. 2005. Gonadotropin-releasing hormone and the control of gonadotrope function. Reproduction Nutrition Development. 45: 243-254.

7 Davidoff M.S., Middendorff R., Müller D. \& Holstein A.F. 2009. The Neuroendocrine Properties of the Leydig Cells. In: The Neuroendocrine Leydig Cells and their Stem Cell Progenitors, the Pericytes. Berlin: Springer, pp.23-44.

8 Dunshea F.R., Colantoni C., Howard K., McCauley I., Jackson P., Long K.A., Lopaticki S., Nugent E.A., Simons J.A., Walker J. \& Hennessy D.P. 2001. Vaccination of boars with a GnRH vaccine (Improvac) eliminates boar taint and increases growth performance. Journal of Animal Science. 79: 2524-2535.

9 Edwards S.A., Borell E.V. \& Bonneau M. 2009. Guest editorial: Scientific and practical issues associated with piglet castration. Animal. 3(11): 1478-1479.

10 Fang F., Su S., Liu Y., Zhang Y., Pu Y., Zhao X., Li Y., Cao H., Wang J., Zhou J. \& Zhang X. 2013. Immunization against recombinant GnRH-I alters ultrastructure of gonadotropin cell in an experimental boar model. Reproductive Biology and Endocrinology. 11: 63-66.

11 Fuchs T., Thun R., Parvizi N., Nathues H., Koehrmann A., Andrews S., Brock F., Klein G., Sudhaus N. \& Beilage E.G. 2009. Effect of a gonadotropin-releasing factor vaccine on follicle-stimulating hormone and luteinizing hormone concentrations and on the development of testicles and the expression of boar taint in male pigs. Theriogenology. 72: 672-680.

12 Haider S.G. 2004. Cell biology of Leydig cells in the testis. International Review of Cytology. 233: 181-241.

13 Hansson K.E., Lundström K., Fjelkner-Modig S. \& Persson J. 1980. The importance of androstenone and skatole for boar taint. Swedish Journal of Agricultural Research. 10(4): 167-173.

14 Hedger M.P. 2015. The Immunophysiology of Male Reproduction. In: Plant T.M. \& Zeleznik A.J. (Eds). Knobil and Neill's Physiology of Reproduction. 4th edn. Amsterdam: Academic Press, pp.805-894.

15 Hemonic A. \& Courboulay V. 2009. Evaluation of the safety, efficacy and production benefits of vaccination against boar taint in male pigs raised under commercial field conditions in France. Revue de Médecine Vétérinaire. 160(8-9): 383-393.

16 Howroyd P., Hoyle-Thacker R., Lyght O., Williams D. \& Kleymenova E. 2005. Morphology of the fetal rat testis preserved in different fixatives. Toxicologic Pathology. 33(2): 300-304.

17 Metz C., Hohl K., Waidelich S., Drochner W. \& Claus R. 2002. Active immunization of boars against GnRH at an early age: consequences for testicular function, boar taint accumulation and N-retention. Livestock Production Science. 74: 147-157.

18 Pauly C., Spring P., O’Doherty J.V., Kragten S.A. \& Bee G. 2009. Growth performance, carcass characteristics and meat quality of group-penned surgically castrated, immunocastrated (Improvac $®$ ) and entire male pigs and individually penned entire male pigs. Animal. 3(7): 1057-1066.

19 Pissinatti A., Burity C.H.F. \& Mandarim-De-Lacerda C.A. 2003. Stereology of the myocardium in Leontophitecus (Lesson, 1840) in Callitrichidae - primates. Journal of Medical Primatology. 32(3): 139-147.

20 Slaoui M. \& Fiette L. 2011. Histopathology procedures: from tissue sampling to histopathological evaluation. Methods in Molecular Biology. 691: 69-82.

21 Stanislaus D., Pinter J.H., Janovick J.A. \& Conn P.M. 1998. Mechanisms mediating multiple physiological responses to gonadotropin-releasing hormone. Molecular and Cellular Endocrinology. 144: 1-10. 
22 Stojanovic S., Uscebrka G., Zikic D. \& Kanacki Z. 2013. Skeletal muscle characteristics of broiler chickens under modified incubation factors. Avian Biology Research. 6(4): 281-288.

23 Tajet H., Andresen O. \& Meuwissen T.E. 2006. Estimation of genetic parameters for boar taint: skatole and androstenone and their correlations with sexual maturation. Acta Veterinaria Scandinavica. 48(Suppl 1): 22-23.

24 Taylor A.A. \& Weary D.M. 2000. Vocal responses of piglets to castration: identifying procedural sources of pain. Applied Animal Behaviour Science. 70(1): 17-26.

25 Thun R., Gajewski Z. \& Janett F. 2006. Castration in male pigs: techniques and animal welfare issues. Journal of Physiology and Pharmacology. 57(Suppl 8): 189-194.

26 Turkstra J.A. \& Meloen R.H. 2006. Active immunisation against gonadotropin-releasing hormone, an active tool to block the fertility axis in mammals. Veterinary Sciences Tomorrow. Disponível em: <http://www.vetscite.org/2006/11/ page/2/>. [Accessed online in January 2017].

27 Turkstra J.A., Zeng X.Y., Van Diepen J.T.M., Jongbloed A.W., Oonk H.B., Van de Wiel D.F.M. \& Meloen R.H. 2002. Performance of male pigs immunized against $\mathrm{GnRH}$ is related to the time of onset of biological response. Journal of Animal Science. 80: 2953-2959.

28 Uscebrka G., Stojanovic S., Zikic D. \& Kanacki Z. 2010. Morphodynamics of embryonic development of skeletal musculature of broiler and layer chickens. Avian Biology Research. 3(4): 179-186.

29 Waites G.M.H., Speight A.C. \& Jenkins N. 1985. The functional maturation of the Sertoli cell and Leydig cell in the mammalian testis. Journal of Reproduction and Fertility. 75: 317-326.

30 Xue J.L., Dial G.D. \& Pettigrew J. 1997. Performance, carcass, and meat quality advantages of boars over barrows: A literature review. Swine Health and Production. 5(1): 21-28.

31 Zamaratskaia G., Andersson H.K., Chen G., Andersson K., Madej A. \& Lundström K. 2008. Effect of gonadotropin-releasing hormone vaccine (Improvac) on steroid hormones, boar taint compounds and performance in entire male pigs. Reproduction in Domestic Animals. 43(3): 351-359.

32 Zamaratskaia G. \& Squires E.J. 2009. Biochemical, nutritional and genetic effects on boar taint in entire male pigs. Animal. 3(11): 1508-1521.

33 Zeng X.Y., Turkstra J.A., Meloen R.H., Liu X.Y., Chen F.Q., Schaaper W.M.M., Oonk H.B., Guo D.Z. \& Wiel D.F.M. 2002. Active immunization against gonadotrophin-releasing hormone in Chinese male pigs: effects of dose on antibody titer, hormone levels and sexual development. Animal Reproduction Science. 70: 223-233. 